RüBELT: Das kann aber doch grundsätzlich von dem Mineralölprodukt abhängen, da Rohöle keinen so dünnen Film bilden werden, wie z. B. Diesel- oder leichtes Heizöl.

BrokkER: Doch, Rohöl kann einen sehr dünnen Film bilden! Man verwechselt das aber öfters mit einer ganz anderen Sache: Wenn die Ballast-Tanks von Tankern gereinigt werden. Hier ist viel Schlamm anwesend mit Eisenoxid und vielen festen Paraffnen; wenn derselbe herausgespült wird, kann die entstandene dicke Masse sich nicht mehr ausbreiten.

Weichart: Ich wollte noch zu der Frage von Herrn Lüdemann eine Antwort geben: An ein Absinken des Oles ist meines Erachtens nicht zu denken, denn es ist ja keiner von den Kohlenwasserstoffen schwerer als Wasser. Wenn das Oil an der Oberfläche nicht mehr zu sehen ist, dann ist es entweder in sehr dünner Schicht vorhanden oder schon ganz im Wasser aufgelöst.

LÜDEMANN: Ich glaube auch nicht, daß es in solchen Massen absinkt. In Berlin war ein Gutachten zu erstellen, und ich ging mit einem langen Bootshaken nach unten. Zunächst bildete sich ein richtiger Olfilm, und die Stange war dick mit Ol beschmiert. Wir wußten nicht, wo es herkam.

RüBeLT: Vielleicht war das Öl an Feststoffe gebunden worden, die bedeutend schwerer als Wasser sind.

GuNkrL: Im Hudsonriver bei New York soll sich auf dem Grund eine ca. $50 \mathrm{~cm}$ starke "Asphaltdecke" gebildet haben. Es soll dort schwierig sein, mit dem Bodengreifer eine Probe zu bekommen, da er kaum hineingeht und noch schwerer wieder herauszuziehen ist.

KüHL: Bei unseren Elbe-Untersuchungen fanden wir die gleiche Situation: Inmitten der Fahrrinne eine feste, etwa $2 \mathrm{~cm}$ dicke, asphaltähnliche Kleischicht, die völlig azoisch war; lediglich leere Muschelschalen wurden darin angetroffen. Der Greifer brachte ebenfalls nur Brocken herauf.

Reuter: Aber nicht bei Cuxhaven!

KüHL: Das stimmt! Es war in der Fahrinne bei Glückstadt-Stadersand bis fast nach Hamburg hinauf.

VAUK: Bei der Erfassung von Ólpestrerlusten ist es für uns von Interesse, zu erfahren, ob und wann ölverschmierte Vögel absinken und sich damit einer Zählung entziehen. Mir scheint nach Beobachtungen und tastenden Versuchen in dieser Richtung wahrscheinlich, daß sich Feststoffe an total verölte Vögel hängen, was schließlich zu einem schlagartigen und endgültigen Absinken der Leiche führt.

SсHÖвERL: Laborversuche in 1-Liter-Flaschen mit im Juni oder Juli geschöpftem Elbwasser das Elbwasser ist in diesen Monaten besonders reichhaltig an ölabbauenden Organismen - haben gezeigt, daß eine auf dem Wasser ausgebreitete, sehr dünne Rohölschicht schon nach einem Tag, ja bereits nach einer Nacht vollkommen perforiert ist. Man kann beobachten, daß schwarze, detritusartige Flodken absinken. Unter dem Mikroskop erkennt man, daß der Niederschlag aus Oltröpfchen besteht, die eine Unmenge Mikroorganismen auf ihrer Oberfläche adsorbiert haben. Könnte dies nicht der Fall gewesen sein? Das Ol wird auf Grund der Belastung durch die Organismen spezifisch schwerer und sinkt dann allmählich auf den Grund. LüDEMANN: Das ist durchaus denkbar.

SCHÖвERL: Denn so entstehen ja sicherlich diese Bitumenablagerungen in den Flüssen.

\title{
Verwendung von Ölschlengeln und Preßluftschleiern zur Eindämmung von Verölungen und Einsatzmöglichkeiten von Ölabschöpfgeräten
}

REUTER: Ich möchte zur Praxis zurïkkommen. Wir haben erfahren, daß man mit Emulgatoren sehr vorsichtig sein soll. Olbindemittel, sdhwerer oder leichter als Wasser, lassen sich nach dem übereinstimmenden Urteil der Nautiker und sonstiger Praktiker in den bei solchen 
beträchtlichen Olverschmutzungen notwendigen großen Mengen weder einbringen noch abfischen. Des öfteren wird die Verwendung von Ölschlengeln angeregt, das sind schwimmende Holz-, Stahl- oder Kunststoffkonstruktionen, die in Strom- oder Windlee der "Ólquelle" ausgelegt werden und das $O \mathrm{Ol}$ bis zur Beseitigung durch Abschöpfen zusammenhalten sollen. Es leuchtet ein, welche Abmessungen ein solcher Olschlengel haben müßte, wenn man derartige Olmengen auffangen wollte. Hinzu kommt, daß "Olquellen" in den havarierten Tankern gerade dann besonders fließen werden, wenn starker Seegang herrscht. Dabei haben wir dann die bekannte Orbitalbewegung des Wassers in den Wellen, die das OOl, das ja dann in einer stärkeren Schicht auf dem bewegten Wasser schwimmt, unter den Schlengeln hindurchzieht, oder diese müssen so tief ins Wasser geführt werden, daß sie nicht mehr zu halten sind. Olschlengel sind also brauchbar im abgesperrten oder absperrbaren Hafenbecken, d. h. im Stillwasser. Noch besser sind Olsperren in Form des auf dem Gewässergrund verlegten Preßluftschlauches, der einen Preßluftschleier hochläßt. Das gilt besonders für Ölhäfen und andere Häfen, vielleicht auch für Seen. Ich stelle mir die Frage: Was können wir tun, wenn ein solcher Unfall von den Ausmaßen der "Torrey Canyon" oder "Anne Mildred Brøvig" hier oder auf der Themse, vor Brunsbüttelkoog oder in der Jade sich ereignen würde? Das kann jeden Tag geschehen! Wollen wir auch dann Emulgatoren einsetzen? Ich kann es mir kaum vorstellen! Bleiben wir bei den OIschlengeln. Kennen Sie eine Konstruktion, die es erlaubt, bei diesen Strömungsgeschwindigkeiten von 1 bis $11 / 2 \mathrm{~m} / \mathrm{sec}$ bzw. im. Seegang der Nordsee, ausgelegt zu werden, und die es dazu auch noch gestattet, das $\mathrm{O} 1$ festzuhalten? Ich frage, ob jemand andere und bessere Erfahrungen gesammelt hat?

I do not believe, that booms have any success in the North Sea or in the open Ocean at an accident like the "Torrey Canyon" or in a river, where the speed of the current exceeds 1 or $2 \mathrm{~m} / \mathrm{sec}$.

Brockis: I agree substantially with that. Experience in Cornwall would suggest so.

Feverhake: Im Hamburger Hafen sind bereits in den dreißiger Jahren Versuche mit sogenannten "Schlengeln" unternommen worden. Diese bestehen aus aneinandergereihten Gliedern von doppelten Stahlrohren, bei denen die offenen Stellen zwischen den Gliedern durch ins Wasser tauchende Blechschürzen überbrückt werden. Die "Schlengel", die noch vorhanden sind, waren ursprünglich für die Abgrenzung von Olbränden gedacht. Bei den Versuchen hat sich ergeben, daß der Olfilm schon bei einer Strömungsgeschwindigkeit des Wassers von etwa $0,10 \mathrm{~m} / \mathrm{sec}$ unter den Schlengeln durchtaucht.

BLOKKER: Ich meine, daß es $1 / 2 \mathrm{~m} / \mathrm{sec}$ ist.

Feuerhake: Nein, $0,30 \mathrm{~m}$ pro Sekunde sind es im Mittel der Preßluft-Olsperre. Diese besteht aus einer Luftblasenwand im Wasser, die von einem Schlauch mit feinen Dïsen auf der Hafensohle durch Einblasen von Druckluft erzeugt wird. Die aufsteigenden Blasen bilden auf der Wasseroberfläche einen kleinen Wasserball, der das Verdriften der Ölschicht verhindert. Bei stark strömendem Wasser funktioniert das, wie Herr REUTER schon sagte, nicht, darüber sind systematische Versuche durchgeführt worden (STEHR 1959, 1964).

Brockrs: In the context of faster flowing rivers, it may be possible, and worthwhile in some circumstances, to use some form of boom which, even if it were to allow some oil to get through, and even if it were to disintegrate in a comparatively short time, may stay in position long enough to allow some clear-up of oil above the boom using other equipment. But I do not believe that the boom by itself is the entire answer to the question.

GUNKEL: Would it not be possible to use a plastic tubing half filled with water and half inflated with air with a fairly wide diameter?

Brockrs: We are in fact looking for a new design rather along the lines which Dr. GunkeI has just suggested. Our search may or may not be successful; it is too early to give any definite information at this stage.

Hellmann: Mir ist folgendes aufgefallen: Bei all diesen Oibekämpfungsmitteln, hauptsächlich bei der Ölpreßluftsperre, wird nie daran gedacht, wie dick die Ölschicht sein darf, wenn man sie mit Aussicht auf Erfolg überhaupt noch bekämpfen will. Herr Blokker hat ja OI- 
ausbreitungsversuche gemacht, und ich glaube, wenn eine bestimmte Schichtdicke unterschritten wird, ist es sinnlos, mit Hilfe eines Ölbekämpfungsmittels das Ol überhaupt noch bekämpfen zu wollen. Und diese Dicke müßte man meines Erachtens auch einmal in den Griff bekommen. Man kann mit Olabschöpfgeräten nicht wirtschaftlich arbeiten, wenn der Olfilm z. B. ein Ausmaß von $0,005 \mathrm{~mm}$ oder weniger aufweist. Das wird nie bedacht. Man bekämptt mit Emulgatoren, man schöpft $a b$, wo nichts mehr abzuschöpfen ist, man baut Preßluftsperren auf, wo der Olfilm schon unter $10 \mu$ liegt usw.

Gunkel: Abschließend, mit Olschlengeln ist im Moment also nicht viel zu machen.

REUTER: Unter gewissen Bedingungen schon, in Hafenbecken, in Stillwasser, im Bodensee, aber wohl nicht auf der hohen See und in stark strömenden Tidegewässern.

Feutrhake: Es gibt auch über die Olausbreitung und die Olabschöpfung in der Literatur Angaben, u. a. von STEHR, die sich aber auf relativ ruhiges Wasser beziehen, nicht auf die offene See.

BLOKKER: Ja, und sie stimmen gut überein mit Experimenten, die ich durchgeführt und publiziert habe.

GUNKEL: I recently heard that there are already special boats in operation, which collect oil from the water surface. Would anybody care to comment on this?

Brockis: There are a number of vessels operating in this way. One is a vessel designed by Shell, Den Haag, and is called the "Wasserwischer". It operates with scoops on both sides of the ship which form a V between the scoop and the side of the vessel. As the vessel moves forward the oil becomes concentrated in the apex of the $\mathrm{V}$ and may be pumped on board.

Feueriake: Im Hamburger Hafen arbeitet ständig und mit gutem Erfolg das „Hamburger Olabschöpfgerät $t^{“}$.

Gunkel: How fast can the ship mentioned by Dr. Brockis go? As I understand it, the system works like a moving boom. Does this not raise a problem similar to the one discussed in regard to the speed of the water current?

Brockis: With the scoops in use I believe the speed is about 2 knots. The vessel has not been designed for use on the open sea and would be of practical value only for small spillages; it would not be of much use at a "Torrey Canyon" scale disaster. Another vessel - built for BP at Hamburg, originally tested there and more recently tested in Britain - works on a different basis. The principle used in this vessel, which has been named "Sea Sweeper" (Fig. D1), is that oil will stick to almost anything preferentially to water. This principle is utilized in the form of a series of moving endless bands which dip into the oil-contaminated water. The oil sticks to the bands and is removed by a scraping device on board of the vessel. The "Sea Sweeper" is the prototype vessel. The individual moving belts projecting at the front are clearly visible. The recent tests carried out by $\mathrm{BP}$ in Britain have shown that it is possible to pick up oil from the surface of a harbour at somewhere between 200 and 300 gallons per hour. A current problem is that the vessel is slow moving and, as it chases the oil, the oil tends to move away. If oil could be propelled towards the moving belts, pick-up rates of 1.000 gallons or more per hour might be achieved.

Feverhake: Es gibt eine große Anzahl von Olabschöpfgeräten, die mehr oder minder wirkungsvoll arbeiten; wir haben im Hamburger Hafen einige des Typs „Hamburger Olabschöpfgerät" in Betrieb. Monatlich sind zwischen 6 und 12 Olverschmutzungen $z u$ verzeichnen; das sind relativ kleine Verschmutzungen, bei denen es sich um Ollachen von geringer Stärke und einer Ausdehnung von einigen 100 bis zu einigen $1000 \mathrm{qm}$ handelt. Das eigentliche hier zur Debarte stehende Problem stellen ja die großen Olverschmutzungen dar. Alle diese Abschöpfgeräte sind nicht mehr bei Verschmutzungen auf offener See oder selbst in ruhigerem Wasser bei solchen mit einigen tausend und mehr Tonnen $\mathrm{O} l$ zu gebrauchen.

SCHWEISFURTH: Ist es möglich, diese Olmengen von Oberflächen „abzuschnüffeln“, wie man es von Badeanstalten kennt, und zwar mit einem Absauggerät, das auf der Wasseroberfläche schwimmt und wie ein Staubsauger arbeitet? 
FEUERHAKE: Ja, etwas Ahnliches stellt das „Hamburger Olabschöpfgerät (System Schuback) dar. Es arbeitet freilich nicht wie ein Staubsauger. Das Gerät gestattet durch Regulieren der Förderpumpe eine Anpassung an die jeweils vorhandene Olschichtstärke. Ober eine sich automatisch einstellende Klappe oder Einlaufrinne nimmt es dicht unter der Wasseroberfläche den Olfilm $a b$ und fördert ihn in einen Behälter. Von diesem Behälter aus wird das Ol abgepumpt, und auf diese Weise wird die mitzufördernde Wassermenge auf ein Minimum beschränkt (vgl. SteHr 1959, 1960, 1963, 1964a, b, c, Kroslewski 1966, HinRichsen 1965).

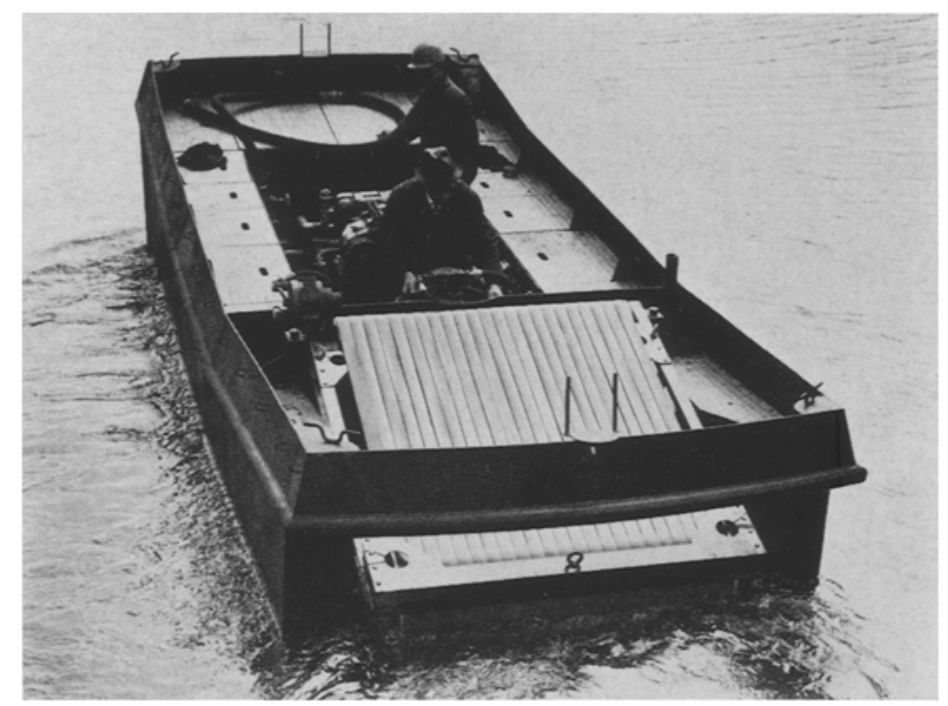

Fig. D1: The Sea Sweeper. A self-propeled oil recovery vessel, incorporating several aspects of a patent recently granted to $\mathrm{BP}$, has been designed and built in Germany by HarmstorF, Wasserbau G. m. b. H. of Hamburg (BP Foto)

SCHWEISFURTH: Wenn man eine Pipette in eine Olschicht sticht, die auf dem Wasser schwimmt, dann kann man das $\mathrm{Ol}$ ja auch absaugen.

FEUERHAKE: Natürlich ist so etwas auch möglich, aber dies gilt nur für Verschmutzungen kleinen Ausmaßes, die nie ein ernstes Problem darstellen.

SchwersfurTh: Warum geht das nicht in größerem Maßstab?

REuTER: Hoher Seegang zum Beispiel wäre hinderlich. In der Nordsee ist jedoch fast nie ruhige See.

Schweisfurth: Der Sauger würde ja auf den Wellen mitschwimmen.

REUTER: Aber Sie dürfen nicht vergessen, daß Sie in der offenen See die Orbitalbewegung der Wellen haben. Auch ohne Einwirkung von Emulgatoren wird bei starkem Seegang alles durcheinandergerissen. Ich komme zurück auf die Erfahrungen mit der "Anne Mildred Brøvig". Havarierte Tanker, ich glaube, das gilt auch für "Torrey Canyon“, geben in erster Linie Ol $a b$, wenn sie sich stark bewegen. Als dieses Schiff ruhig lag, gab es praktisch gar kein Ol ab. Ein solcher Tanker hat ja nicht so große freie Laderäume wie ein Stück- oder Massengutfrachter. Die „Anne Mildred Brøvig“ z. B. besaß rund 30 einzelne in sich abgeschlossene Tanks mit je etwa 1000 bis $25000 \mathrm{t}$ Inhalt. Wenn etwas passiert, z. B. die "Torrey Canyon" stößt auf die „Seven Rods", dann tritt zunächst eine begrenzte Menge Ol aus den Tanks aus, die direkt verletzt worden sind. So ist es auch bei der „Anne Mildred Brøvig" gewesen; da, wo die „Pentland“, der Kollisionsgegner, in die zwei letzten Tanks auf der Backbordseite hineingefahren ist, ist das OI ausgelaufen, und dort und in dem ebenfalls beschädigten achteren 
Maschinenraum hat das Schiff einige Streben getrennt. Alle anderen Teile sind erst einmal heilgeblieben! Wer im Krieg bei der Marine war und einmal eine Tankerversenkung miterlebt hat, der weiß, welch zähes Leben diese Tanker haben. Um die Tanker, wenn sie nicht gleich explodiert waren, zu versenken, mußten sie „entlüftet" werden; so, glaube ich, hieß der terminus technicus bei den Kriegern damals. Ich komme darauf zurïck. Wenn also der Tanker nach diesem ersten "Generaleingriff ", wo er vielleicht 3000 bis 4000 t OOl verliert, weiteres ôl abgeben muß3, geschieht das einmal durch die bei der Revierfahrt offenen Lüfter. Die Lüfter wird man aber meist durch Taucher wieder schließen können. Wenn ein solches Schiff ganz auseinanderbrechen würde, dann würde wiederum eine begrenzte Menge an Ol frei. Das sind dann aber Verhältnisse, bei denen starker Seegang herrschen wird, und dann können Sie mit allen genannten Mitteln nichts anfangen.

SCHWEISFurTh: Es besteht aber doch eine Möglichkeit, wenn sich ein solcher Sturm legt; dann könnte die Situation, bevor Ol die Küste erreicht, mit Schnüffelgeräten harmonisiert werden.

ReUteR: Ich glaube, das ist sehr theoretisch, weil inzwischen das Ol sehr weit verteilt ist. Wenn Sie überlegen, daß ein Sturm dieser Stärke mit etwa $60 \mathrm{~km} / \mathrm{h} 24$ Stunden lang weht, dann sind das schon ca. $1500 \mathrm{~km}$. Das Ol verdriftet mit etwa $4 \%$ der Windgeschwindigkeit, das wären also schon $60 \mathrm{~km}$. Das ist ungefähr der Abstand gewesen, den „Anne Mildred Brøvig" von Borkum gehabt hat. Obwohl wir mindestens eine Wodhe lang ständig nördliche Winde hatten, ist trotzdem kein Ol weiter südlicher als $8 \mathrm{sm}$ vom Wrack gesichtet worden! Wir sind fast jeden Tag bzw. 2- bis 3 mal wöchentlich über diese Stelle geflogen; wir haben südlicher als $8 \mathrm{sm}$ yom Schiff entfernt nichts feststellen können. Nach Norden hin wurden feinste Filme allerdings wesentlich weiter beobachtet. Woran liegt das? Natürlich herrschen besondere Strömungsverhältnisse in der Deutschen Budt, und zwar entgegen dem Uhrzeigersinn - vielleicht kann Dr. LUCHT dazu noch etwas sagen! Offenbar wirken sich Verdunstung und Bakterienangriff, begünstigt durch die schnelle Verteilung, viel stärker aus, als bisher angenommen. Vielleicht tritt diese theoretische Voraussetzung der Windverdriftung, $4 \%$ der Windgeschwindigkeit, gar nicht so ein, wie wir es befürchten mußten. Was haben wir denn überhaupt an Strandverschmutzungen gefunden? Ein Stück von etwa $10 \mathrm{~km}$ oder $15 \mathrm{~km}$ am Hennestrand in Dänemark, wovon - nach den genommenen Proben - gesagt wird, daß es gar kein Ol der „Anne Mildred Brøvig“ gewesen sei. Darüber kann man vielleicht streiten! Aber zurück zu Ihrer Frage: Sicherlich haben wir nach einer gewissen Zeit, nachdem der Sturm sich gelegt hat, wenn aber noch Dünung da ist, keine markanten, scharfbegrenzten Ớlströme mehr, sondern nur noch feinste, weitverteilte Olfilme. Weiter: Ob Sie nun unsere Schiffe betrachten, die draußen Wache gehalten haben, oder die starken Hochseeschlepper der Holländer, sie können wohl bei nahezu jedem Seegang draußen bleiben. Ein solches Spezialschiff, das muß sidherlich, wie man so schön sagt, "den Schwanz einziehen" bei Windstärken von 6 bis 7; dann fährt es also nach Helgoland. Bis es dann wieder auf See ist - von Helgoland bis zur Unfallstelle waren es etwa $40 \mathrm{sm}$ - das dauert allein für das Schiff ca. 4 Stunden.

ToмсzAк: Ich glaube, man sollte zu den Zweifeln, die Herr Reuter äußerte, kurz Stellung nehmen. Die Winddrift des Öles ist experimentell so klar erforscht, daß meines Erachtens Zweifel daran gar nicht möglich sind (ToмсzAK 1964). Nach Borkum konnte kein Ol kommen, da entgegen der Annahme von Herrn ReUter vom 23. Februar bis 8. März mit Ausnahme einer 20stündigen Periode nordwestlicher Winde am 1. März die Winde immer aus S bis WSW wehten. Die aus dem Wind berechnete Oldrift weist von Anfang an nach NE bis NNE. Die gemäß dieser Rechnung zu erwartenden Strandverschmutzungen liegen bei Blaavands Huk (Fintreffen des Oles am 28. Februar) und bei Loekken in der Jammerbucht (Eintreffen am 8. März). An beiden Stellen ist das Ol an diesen Tagen auch tatsächlich gefunden worden (KOHNKE 1967).

BLOKKER: Am Bodensee verwendet man ebenfalls Abschöpfgeräte. Dort kann man noch etwas machen, wenn es sich nur um einige Tonnen Ol handelt, aber nicht in so einem Falle wie bei dem „Torrey-Canyon"-Unglück.

REUTER: Wer kann solche teuren Geräte vorrätig halten? Es wäre gut, wenn man wenigstens einen Versuch mit einem Schlengel machen könnte; man müßte aber 6, 8, 10 verschiedene 
Schlengeltypen testen. Wir Ingenieure und Nautiker müssen uns da etwas Besseres einfallen lassen. Wenn Sie ferner an die internationalen Schwierigkeiten denken, von denen Herr BrockIs schon sprach, wo er ja doch trotz aller Hoffnung am Ende äußert, praktisch doch auf der Stelle treten zu müssen, weil stets bestimmte Leute Widerstand leisten werden.

LüDEMANN: Ist das Ol nur dort ausgelaufen, wo die Tanks angeknackt waren?

ReUter: Ja, zuerst.

Lüdemann: Das Schiff wird verlassen, geht unter, liegt auf Grund. Die anderen mit Ol gefüllten Tanks sind in Ordnung; wann kommt denn das nun heraus?

REUTER: Wenn die Lüfter offen sind, tritt laufend $O 1$ heraus, und zwar besonders dann, wenn sich das Schiff durch den Seegang stark bewegt. Wenn das Schiff dann nochmals durchbricht, würden wieder mindestens 3 Tanks - es sind meistens 3 Tankreihen in Längsrichtung vorhanden - mit je 1000 bis $2000 \mathrm{t}$ auslaufen. Wir haben damals die Meinung vertreten, das beste wäre, das Schiff ginge in ganzer Länge auf Tiefe. Dann könnten wir abwarten, bis ruhiges Wetter kommt, schließen durch Taucher die Lüfter und dann leichtern wir das Schiff von unten her! Das waren unsere Vorstellungen, nachdem wir uns von den ersten Katastrophengedanken freigemacht hatten. Ich glaube allerdings, dies wäre bei der "Torrey Canyon" nicht möglich gewesen, weil sie sehr schnell auseinanderbrach. Im übrigen sind bei der "Anne Mildred Brøvig" etwa 15000 t Ol ausgelaufen; höchstens 2000 bis 3000 t Oll ließen sich davon mit Emulgatoren bekämpfen!

GunkEL: Wir hatten zur Zeit des Unfalls der „Anne Mildred Brøving“ bei Helgoland ein Oltreiben. Es handelte sich um braune, teerige Klumpen, welche wir untersuchen ließen. Es wurde uns dann mitgeteilt, es handele sich um ein bestimmtes Schmieröl, das auf keinem Fall auf dem Schiff gewesen sei. Ich könnte mir vorstellen, daß sich manche Kapitäne die Gelegenheit nicht haben entgehen lassen, Ol loszuwerden, unter der Annahme, daß es doch auf den Unfall der „Anne Mildred Brøvig" zurückfällt.

Reuter: Wir haben in jenen Tagen häufig die Nordsee überflogen. Es war außergewöhnlich interessant, die Verseuchung der inneren Deutschen Bucht mit Ol von oben zu sehen. Id habe den Piloten des Marineflugzeuges gefragt: „Ist das hier immer so, oder nutzen jetzt irgendwelche Leute die Gelegenheit aus, um Schmutzöl über Bord zu pumpen?" Er entgegnete: „Das ist hier das übliche Bild!" Auf dem Rückflug entdeckten wir vor Wangerooge tatsächlich einen Tanker von etwa 5000 bis $6000 \mathrm{t}$, der eine etwa $2 \mathrm{sm}$ lange Olfahne von etwa $300 \mathrm{~m}$ Breite hinter sich herzog. Leider hatten wir nicht mehr genug Benzin, um herunterzugehen und den Namen abzulesen. Da ist sehr viel gesündigt worden, und es wird laufend weiter gesündigt!

LüDemann: Wir erhalten Ol auf dem Schiffahrtsweg nach Berlin. Dort haben wir große, umfangreiche Tanklager; beim Leichtern geht eine Menge OI in die Havel. Das Fischereiamt in West-Berlin hat Kontrollen vorgenommen, aber dessen Fischereiboot ist natïrlich bekannt. Dieses Olablassen erfolgt nachts. Auf dem Schiffahrtsweg nach West-Berlin passiert das wegen anderer und härterer Bestimmungen nicht. Herr Reuter, was wäre passiert, wenn Sie den Namen des Tankers mit der Olfahne hätten feststellen können?

REUTER: Man muß zuerst prüfen, ob es sich um internationale oder nationale Gewässer handelt. Weiter müssen wir feststellen, ob das Schiff einer Nation angehört, welche die O1konvention unterschrieben hat. Dann kommt das Problem des Beweises. $\mathrm{Da}$ wir keine Probe hätten nehmen können, hätte Aussage gegen Aussage gestanden. Es ist schon schwierig genug, ein an der Pier liegendes Schiff, das $\mathrm{Ol}$ abläßt, zu belangen und zu bestrafen. Aber zu dieser ganzen Frage dürfen Sie einen Bundesbeamten nicht fragen, der ist für die Reinhaltung nicht zuständig, das ist Ländersache! Dazu sollte Herr FeurRhAKe etwas sagen.

FEUERHAKE: Im Hamburger Hafen ist die Zahl der ermittelten Verschmutzer hoch, aber die Strafen (Bußgelder wegen Ordnungswidrigkeit) sind geringfügig. Gesetzlich kann eine Buße bis zu 10000,-DM verhängt werden, die gegen den Verantwortlichen, also den Kapitän oder den Leitenden Ingenieur, ausgesprochen wird. $\mathrm{Ob}$ und wieweit die Reederei für den Betrag einsteht, ist nicht bekannt. Die "Taxe“, $d$. h. die gewöhnlich verhängten Bußen, liegen wesentlich niedriger. Ich habe noch nicht erfahren, daß eine Buße von $10000,-D M$ verhängt worden ist. 
Gunkel: Ich war mit der "Meteor" vor ein paar Jahren im Persischen Golf. Dort herrscht ein starker Tankerverkehr, und an vielen Stellen wird Ol übernommen. Wir haben aber während der ganzen Zeit nicht ein einziges Mal einen Olfilm gesehen. Es wäre interessant, die Ursache hierfür genauer zu untersuchen, denn sicherlich wird von den Tankern zumindest etwas $\mathrm{OI}$ über Bord gegeben bzw. werden sich geringfügige Leckagen in den Olleitungen finden, die dort Ladestationen versorgen. Ich glaube nicht, daß die hohe Wassertemperatur direkt verantwortlich ist, sondern der Verdacht liegt nahe, daß hier ein starker mikrobieller Abbau stattfindet.

ReUTER: Dies kann auch daran liegen, daß die Schiffe vorher auf hoher See Schmutzöl von Bord gegeben haben. Denn wenn es dort in den Hafen kommt, muß es ja sauber sein, sonst bekommt es kein Ol. Vielleicht ist es auch eine Frage der Windverdriftung. Seeleute, die diese Route gefahren sind, behaupten, daß es dort schon $100 \mathrm{sm}$ vor einem Olhafen pestilenzartig stinken würde. Ihre Beobachtung verwundert mich; aber ich bin bei diesem Problem überfragt.

\section{Ölbekämpfung und Bergung des Tankers „Anne Mildred Brøvig“}

REUTER: Zunächst möchte ich darauf hinweisen, daß ein sehr umfangreicher Erfahrungsbericht der Wasser- und Schiffahrtsdirektion Hamburg über diesen. Unfall existiert. Interessenten können ihn bei mir anfordern.

Etwa $40 \mathrm{sm}$ vor Helgoland wurde der 25 000-t-Tanker "Anne Mildred Brøvig“ von dem Frachter „Pentland" gerammt. Die „Anne Mildred Brøvig" geriet in Brand, Ol strömte aus, das auf der Wasseroberfläche ebenfalls in Brand geriet. Durch die Tideströmung vertrieb das Wrack in Richtung Norden und versank bei einer Wassertiefe von $38 \mathrm{~m}$. Ober den Aufbauten befanden sich $22 \mathrm{~m}$ Wasser. Das Schiff hatte 30 Tanks von je 1000 bis 2500 t Inhalt. Größere Olmengen traten insbesondere bei schlechtem Wetter aus, die deutlich sichtbar waren. Teilweise war die Ölschicht hinter dem Tanker unter $1 \mathrm{~mm}$ stark. Von mehreren Schiffen wurden in das austretende OOl verschiedene Emulgatoren hineingespritzt. Insgesamt wurden 740001 Emulgatoren gesprüht, davon 40\% Moltoclar und 40\% Ascal Super 7-11. Außerdem wurden Slix, Gamlen, BP 1002 verwandt. Es waren noch größere Mengen an Emulgatoren vorrätig, die nicht mehr eingesetzt worden sind, nachdem die verhältnismäßig ungünstige Beurteilung dieser Bekämpfung durch Wissenschafler, insbesondere von der Bundesanstalt für Gewässerkunde, bekannt wurde. Außerdem hatten wir bemerkt, daß größere Schäden durch das Ol nicht eintraten. Den Olaustritt aus dem Wrack zeigt Abbildung D2.

Das Wrack der "Anne Mildred Brøvig" wurde mit dem größten Teil des noch in den Tanks befindlichen Ols geborgen. Hierbei hat sich die holländische Firma SMID sehr viel Ruhm erworben, die die schwierige Aufgabe mit sehr viel Geschick, Geduld und Tüchtigkeit löste. Sie hatte die Idee, das Achterschiff, das durch Explosion und Brand nur noch teilweise mit dem Vorschiff zusammenhing, durch eine Reihe von kleinen Hafladungen abzusprengen, um dadurch das Vorschiff auf seiner eigenen Ladung zum Aufschwimmen zu bringen. Vor der Sprengung wurde mit Hilfe des Pumpenschiffes "Gudrun Bonertz" begonnen, das Ladungsöl aus den Tanks in bereitstehende Tankleichrer auszupumpen. Bei ruhiger See wurden Schlauchverbindungen zu den vorderen Tanks hergestellt (Abb. D3). Dieses Pumpmanöver ist jedoch nur etwa bis Windstärke 3 möglich. Trotz der damals für einige Tage herrschenden praktischen Windstille traten Wellen von 40 bis $50 \mathrm{~cm}$ Höhe auf. Die Tideströmung liegt etwa in der Größenordnung von $0,5 \mathrm{~m} / \mathrm{sec}$. In diesem Bereich wïrde ich es, wie schon ausgeführt, für ausgeschlossen halten, mit Olschlengeln zu arbeiten. Die Bergung fand Ende April statt, und wir hatten glücklicherweise 8 bis 10 ruhige Tage. Zunächst wurden durch Taucher die Lüfter geschlossen. Nach der Sprengung schwamm das Vorschiff mit der Restladung auf; es hatte jedoch noch einen Tiefgang von $18 \mathrm{~m}$. Das Achterschiff liegt heute noch an dieser Stelle. Während dieser Arbeiten sind weitere, nicht unbeträchtliche Olmengen freigeworden. Das muß in Kauf genommen werden, wenn man auf diese Art und Weise die Gewässerverschmutzung durch die noch verbliebenen ca. 25000 t Ol vermeiden kann.

Das aufgeschwommene Wrack wurde nach Helgoland geschleppt, wobei auf dem ganzen Weg Preßluft auf die leergepumpten Tanks gedrückt wurde, um ein erneutes Sinken zu vermeiden. 